\title{
Indian Frankincense Oil
}

National Cancer Institute

\section{Source}

National Cancer Institute. Indian Frankincense Oil. NCI Thesaurus. Code C107317.

The oil extracted from the resin of various Boswellia species. Frankincense oil is used in perfumes, cosmetics, and pharmaceuticals. 